\title{
Mobile Living Labs 09: Methods and Tools for Evaluation in the Wild
}

http://mll09.novay.nl

\author{
Henri ter Hofte \\ Novay \\ P.O. Box 589, \\ 7500 AN Enschede \\ The Netherlands \\ Henri.terHofte@novay.nl
}

\author{
Kasper Løvborg Jensen \\ Dept. of Electronic Systems \\ Aalborg University \\ Niels Jernes Vej 12, \\ 9220 Aalborg \\ Denmark \\ klj@es.aau.dk
}

Petteri Nurmi

Helsinki Institute for

Information Technology HIIT

University of Helsinki

P.O. Box 68

FI-00014, Helsinki, Finland

petteri.nurmi@cs.helsinki.fi

\author{
Jon Froehlich \\ DUB Institute \\ Computer Science and \\ Engineering \\ University of Washington \\ Seattle, WA 98195 USA \\ jroehli@cs.washington.edu
}

\begin{abstract}
In a Mobile Living Lab, mobile devices are used to evaluate concepts and prototypes in real-life settings. In other words, the lab is brought to the people. This workshop provides a forum for researchers and practitioners to share experiences and issues with methods and tools for Mobile Living Labs. In particular, we seek to bring together people who have applied methods for Mobile Living Labs and people who build tools for those methods.

The aim of the workshop is twofold. First, to make an up-to-date overview of current methods and tools for conducting user studies in Mobile Living Labs - highlighting their individual strengths and weaknesses. Second, to uncover challenges that are not adequately addressed by current methods and tools and to come up with ideas and requirements that could fill this gap thus serving as beacons for further research and development in this area.
\end{abstract}

\section{Categories and Subject Descriptors}

H.5.2 [Information Systems and Presentation]: User Interfaces - Evaluation/methodology, Graphical user interfaces (GUI), Prototyping, Theory and methods.

\section{General Terms}

Measurement, Performance, Experimentation, Human Factors.

\section{Keywords}

Living Labs, mobile, user experience, field study, in-situ evaluation, methods, tools.

\section{TOPIC}

Mobile devices have become truly ubiquitous computing platforms, offering a wide range of functionality from traditional voice/text communication to GPS navigation and mobile friend finding. Much of their value stems from their ability to be taken anywhere offering quick, convenient access to applications and services in a variety of contexts. These "contexts of use" are even more important now as context-aware applications (such as location-aware services) are becoming a reality in many contemporary smartphones. This context of use, however, makes it difficult for researchers to evaluate mobile device applications and interactions using traditional human-computer interaction methods. Whereas some aspects of the user experience can be evaluated with lab experiments, interviews, focus groups and/or surveys, many other aspects are harder to investigate if taken out of the natural context of use (e.g., out in the wild).

Instead of focusing solely on bringing people to the lab, researchers who want to evaluate mobile devices and services are increasingly doing the opposite: bringing the lab to the people [1]. This is a key ingredient of the "Living Labs" approach [7], which employs a range of methods, including: self-report methods (e.g., experience sampling, diaries, and day reconstruction), measurement (e.g., application usage logging, context logging), as well as observation (e.g., ethnography).

For Mobile Living Labs, smartphones are often used, not only as a platform to deliver new mobile services, but also as a platform for data collection tools. Contemporary smartphones can be utilized to collect all kinds of data (audio, video, user input), according to a wide range of methods. These methods range from automatic data collection without user intervention (logging) to asking users small questions at random moments (experience sampling). Some tools used in Mobile Living Labs recently include ContextPhone [6], MyExperience [1], Xensor [3], RECON [4] and BeTelGeuse [5].

Of course, data gathered by such tools is different from observations by researchers (e.g., well-trained ethnographers), but using smartphones enables studying more persons, longer and at times and locations where observations by researchers would have been difficult.

This workshop provides a forum for researchers and practitioners interested in sharing experiences and issues with methods and tools for Mobile Living Labs. In particular, both people who have applied methods in Mobile Living Labs as well as those who built tools for Mobile Living Labs participate in the workshop. 
Topics and questions addressed in the workshop include:

- When to choose taking the lab to the people instead of taking people to the lab?

- What are the merits and limitations of Mobile Living Lab methods in general?

- For which kind of applications are Mobile Living Labs beneficial? (e.g., only applications that involve social networks and context-aware applications, or are there other categories as well?)

- What are the merits and limitations of Mobile Living Lab evaluation methods in general? What are the merits and limitation using it for formative evaluation (i.e., to inform the design of new applications) and for summative evaluation (i.e., to assess the (user interface of) an application)?

- What are the relative merits and limitations of self-report methods, measurement methods and observation methods for studying various aspects of mobile user experience?

- How should one deal with long-term and large-scale Mobile Living Labs?

- Which tool support is needed for configuring, deploying, data collection and analysis of studies in Mobile Living Labs?

- What are the merits and limitations of using people's own mobile devices versus handing out new devices for a study?

- To what extent can we study mobile user experience with tools embedded in stationary infrastructure?

- What is known about the reliability and validity of these methods and tools? What are the open issues?

- How to deal with informed consent, privacy, data/device theft, loss and corruption?

- What have we learned so far? What have been the big outcomes from previous Mobile Living Lab workshops?

\section{WORKSHOP AGENDA AND RESULTS}

The agenda of the workshop, position papers and a summary of the workshop results are published on the workshop website, http://mll09.novay.nl.

\section{WORKSHOP ORGANIZERS}

- Henri ter Hofte, Novay, The Netherlands

- Kasper Løvborg Jensen, Aalborg University, Denmark

- Petteri Nurmi, Helsinki Institute for Information Technology, Finland.

- Jon Froehlich, University of Washington, USA

\section{PROGRAMME COMMITTEE}

- Tom Broens, Novay, The Netherlands

- Sunny Consolvo, Intel Research, USA

- Alexandre Fleuri, Aalborg University, Denmark

- Anne Marie Kanstrup, Aalborg University, Denmark

- Joke Kort, TNO Information \& Communication Technology, The Netherlands

- James Landay, University of Washington, USA

- Yelena Nakhimovsky, Google, USA

- Ingrid Mulder, Delft University of Technology \& Rotterdam University, The Netherlands

\section{ACKNOWLEDGEMENTS}

This work is supported by the Amsterdam Living Lab project (http://www.amsterdamlivinglab.nl/).

\section{REFERENCES}

[1] Connelly, K.; Siek, K.A.; Mulder, I.; Neely, S.; Stevenson, G.; Kray, C. (2008). Evaluating Pervasive and Ubiquitous Systems, IEEE Pervasive Computing, 7(3), 85-88.

[2] Froehlich, J., Chen, M., Consolvo, S., Harrison, B., \& Landay, J. (2007). MyExperience: A System for In Situ Tracing and Capturing of User Feedback on Mobile Phones. In Proceedings of MobiSys 2007, San Juan, Puerto Rico.

[3] Hofte, G.H. ter (2007). What's that Hot Thing in my Pocket? SocioXensor, a smartphone data collector. In: Proceedings of e-Social Science 2007, the Third International Conference on e-Social Science, October 7-9, 2007, Ann Arbor, MI, USA.

[4] Jensen, K.L. (2009). RECON: Capturing Mobile and Ubiquitous Interaction in Real Contexts. In: Proceedings of MobileHCI 2009, Bonn, Germany.

[5] Kukkonen, J., Lagerspetz, E., Nurmi, P., Andersson, M. (2009). BeTelGeuse: A Platform for Gathering and Processing Situational Data. IEEE Pervasive Computing, 8(2), 49-56, 2009.

[6] Raento, M., Oulasvirta, A., Petit, R., Toivonen, H. (2005). ContextPhone: A Prototyping Platform for Context-Aware Mobile Applications. IEEE Pervasive Computing, 4, 51-59.

[7] Schumacher, J, \& Niitamo, V.P. (Eds). (2008). European Living Labs: A new approach for human-centric regional innovation. Wissenschaftliger Verlag, Berlin, Germany. 